\title{
Detección de la Depresión mediante cuestionarios: Comparación de una sola pregunta con los datos de la entrevista en una muestra de adultos de edad avanzada
}

\section{Mallon ${ }^{\star \star \star \star}$ \\ J. Hetta $a^{\star \star \star \star *}$}

* Unidad del Sueño, Departamento de

Neurociencias, Psiquiatría, Hospital

Universitario, SE-751 85 Uppsala

** Servicio de Psiquiatría. Hospital Falun, SE-791 82 Falun

*** Instituto Clínico de Neurociencias, Servicio de Psiquiatría, SU/Sahlgrenska, SE413 45 Göteborg

SUECIA

RESUMEN - El objetivo de este estudio fue el de determinar qué relación existía entre una pregunta sobre el humor depresivo, incluida en un cuestionario, y el diagnóstico de depresión establecido según los criterios DSM-III-R. Se envió un cuestionario por correo a una muestra de personas con edades comprendidas entre los 57 y 79 años, elegida al azar en la población del Condado de Dalama, en Suecia central. La proporción de respuestas fue del $74,1 \%$ (1.328 personas). Todos aquellos individuos que, en el cuestionario, habían admitido estar deprimidos fueron seleccionados para una entrevista, de ellos, 28 aceptaron participar (el 74\% de los que habían sido elegidos).

En el momento de ser entrevistadas, 21 personas (el 75\%) padecían trastornos afectivos según los criterios DSM-III-R o estaban bajo tratamiento debido a su depresión. 12 personas (el 42,9\%) padecían depresión mayor o estaban bajo tratamiento. Asimismo, 9 individuos (el 32,1\%) padecían distimia. Incluso los niveles moderados de depresión fueron relacionados con una incapacidad funcional.

Una sola pregunta sobre el humor depresivo supone un buen punto de referencia y aunque una respuesta afirmativa no puede considerarse como decisiva a la hora de llegar a un diagnóstico, ésta merece un estudio más profundo. 


\section{Introducción}

La depresión está considerada como un problema de salud pública muy importante y su estudio forma parte integral de las investigaciones sobre el bienestar y la salud de la personas de edad avanzada. Varios trabajos han demostrado que los síntomas depresivos están relacionados con una salud precaria y con una incapacidad funcional (Valliant et al. 1996, Gurland, 1976, Kivelä et al. 1988). Estos estudios también indican que la depresión mayor tiene que ver con el aumento de la mortalidad en personas mayores (Valliant et al. 1996, Murphy et al. 1988).

Las proporciones de incidencia de la depresión varían considerablemente y aún no es posible determinar si aumentarán con el paso de los años (Palsson \& Skoog 1997, Kanowski 1994, Roberts et al. 1997, Beekman et al. 1999). Según los estudios realizados en una comunidad determinada, los índices de prevalencia oscilan entre el $1 \%$ y el $17 \%$ en personas mayores. Los estudios que utilizan la descripción que los pacientes realizan de sus propios síntomas como instrumento de investigación, suelen obtener índices de prevalencia más altos que aquellos que se sirven de entrevistas estructuradas y criterios estrictos (Kanowski 1994, Beekman et al. 1999, Skoog et al. 1993). Uno de los posibles argumentos que se han ofrecido para explicar la diferencia en la prevalencia es que los síntomas depresivos transitorios son normales en adultos de edad avanzada aunque probablemente no serían clasificados como trastornos depresivos (Gurland 1976, Blazer et al. 1987, Henderson et al. 1993).

La razón fundamental por la que se investiga la depresión no es otra que la de ofrecer un método económico para detectar casos potenciales de esta enfermedad en la población general. Con el fin de valorar la depresión en personas de edad avanzada es necesario recurrir a un instrumento de investigación que sea muy fácil de utilizar y que permita superar los problemas específicos de las personas mayores. Tanto la brevedad como la sencillez pueden ser dos aspectos fundamentales en el caso de que los inventarios de autoevaluación supongan un problema para personas de edad avanzada. Es posible que las preguntas les resulten difíciles porque no están familiarizados con escalas de elección múltiple, porque no puedan entender o interpretar las preguntas (Knäuper \& Wittchen 1994) o simplemente porque se cansan cuando tienen que completar el cuestionario (Irwin et al. 1999).

En un estudio que estaba centrado en la utilidad clínica de los instrumentos que sirven para investigar la depresión, Schade (Schade et al. 1998) descubrió que aquellos que son breves funcionan tan bien como los cuestionarios largos en la población general. Por esta razón, se ha intentado buscar alternativas con menos items para las personas de edad avanzada (Almeida \& Almeida 1999, Hoyl et al. 1999, Allen et al. 1994, Weyerer et al. 1999, Andersen et al. 1994).

El objetivo principal de este estudio fue el de investigar qué relación existía entre una pregunta sobre la depresión incluida en un cuestionario, y el diagnóstico establecido según los criterios DSM (APA 1987) basados en la entrevista. También se obtuvo información sobre las características demográficas, la gravedad de la depresión y el nivel de funcionamiento en lo que respecta a la autoevaluación del humor depresivo. 


\section{Material y métodos}

\section{Material}

Se eligió al azar una muestra de 1.792 personas con edades comprendidas entre 57 y 79 años que vivían en Condado de Dalarna, en Suecia Central. En 1995, se envió por correo un cuestionario a todas estas personas y respondieron el $74,1 \%$. De las 1.328 personas que respondieron, 623 (el 46,9\%) eran hombres y 705 (el 53,1\%) mujeres. La edad media era de 67,8 +/- 5,9 años. Además, el 14,2\% de estas personas no estaban casadas (eran solteras, estaban divorciadas, separadas o viudas) y el 14,0\% vivían solas.

\section{Métodos}

El estudio fue llevado a cabo en dos fases. En la primera fase, se analizó la el humor depresivo a través de una pregunta incluida en el cuestionario que fue enviado por correo. En la segunda fase, se entrevistó a aquellos individuos que, en el cuestionario, habían admitido estar deprimidos.

\section{A. El Cuestionario}

El cuestionario "Sueño y Salud" estaba formado por 89 preguntas que comprendían variables demográficas, hábitos de sueño, insomnio, enfermedades médicas, síntomas de depresión y ansiedad y medicaciones.

El humor depresivo fue diagnosticado mediante una sola pregunta: ¿Qué dificultades implican para usted un bajo estado de ánimo (depresión)? que tendría que ser contestada utilizando una escala de cinco puntos $(1=$ ningún problema, $2=$ problemas leves, $3=$ problemas de cierta importancia, $4=$ problemas graves, $5=$ problemas muy graves). Se consideró que aquellas personas cuyas puntuaciones fueron de 4 y 5 estaban deprimidas.

\section{B. Entrevistas clínicas}

En la segunda fase, se invitó a participar en las entrevistas a las personas que, en el cuestionario, habían admitido padecer depresión. El primer contacto se realizó por medio de una carta,en la que se explicaba el propósito de la entrevista, y a la que siguió, pasados varios días, una llamada telefónica. Un psiquiatra llevó a cabo las entrevistas seis meses después de que se hubieran devuelto los cuestionarios por correo. Las entrevistas se realizaron en casa de las personas seleccionadas o en la consulta de su médico de familia.

1. Valoración de las alteraciones psiquiátricas

Con el fin de llegar a un diagnóstico siguiendo los criterios DSM-III-R (APA 1987), se utilizó la entrevista clínica semiestructurada para DSM-III-R (SCID) (Spitzer et al. 1992). La SCID se utiliza para hacer un diagnóstico en el Eje I y lo administran médicos o profesionales formados en salud mental que estén familiarizados con la clasificación DSM-III-R y con los criterios de diagnóstico. Junto con los criterios DSM-III-R que se utilizan para diagnosticar una depresión mayor y una distimia, se admitía que una depresión estaba siendo tratada si los pacientes tomaban antidepresivos bajo prescripción médica y si el diagnóstico había sido realizado por un médico. Se preguntó a los pacientes si anteriormente habían tomado antidepresivos debido a su enfermedad. También se les preguntó si algún familiar directo había sufrido depresiones o cualquier otra enfermedad psiquiátrica.

2. Valoración de la gravedad de la depresión

El entrevistador utilizó la Escala de Valoración de la Depresión de MontgomeryAsberg (MADRS) (Montgomery \& Asberg 
1979). Se trata de una escala de valoración, utilizada sólo por expertos, que evalúa la gravedad de una depresión. Consiste en diez preguntas sobre los síntomas comunes de esta enfermedad que han de ser clasificadas según una escala de seis puntos.

Al final de la entrevista, se entregó la Escala de Depresión y Ansiedad Hospitalarias (HAD) a los pacientes, pidiéndoles que la rellenaran en un periodo no superior a dos semanas y que la devolvieran por correo. Por tanto, las valoraciones obtenidas según la MADRS, así como las de la escala HAD se realizaron en ocasiones diferentes. La escala HAD (Zigmond \& Snaith 1983) es un instrumento de autoevaluación según el cual, la gravedad de la ansiedad y de la depresión se calcula atendiendo a una escala de cuatro puntos (de 0 a 3 ). Hay siete preguntas relacionadas con la depresión (HADD) y siete con la ansiedad (HAD-A), ambas con un rango de puntuación de 0-21. Zigmond y Snaith han recomendado dos puntos de corte, es decir, ocho para los casos posibles y 11 para los definitivos. Aquellos items que están relacionados con los síntomas somáticos de la depresión y la ansiedad no están incluidos en la escala HAD.

\section{Valoración del funcionamiento}

Se estableció el nivel total de funcionamiento del paciente utilizando la Escala de Valoración Global de Funcionamiento (GAF) (Eje V en DSM-III-R) (APA 1987). La escala GAF (puntuación de 1 a 100) evalúa el funcionamiento psicológico, social y ocupacional pero no incluye las discapacidades en el funcionamiento del individuo debido a limitaciones físicas. La valoración se llevó a cabo utilizando el nivel más alto de funcionamiento en el mes anterior.

El protocolo de estudio fue aprobado por el Comité Ético de la Facultad de Medicina de la Universidad de Uppsala en Suecia.

\section{Análisis estadístico}

Los análisis estadísticos se realizaron mediante un ordenador Macintosh, utilizando el programa de análisis estadístico SPSS 6.1 (SPSS Inc., Chicago, IL, USA). Se utilizaron los métodos estándar para calcular los valores medios así como las desviaciones. Cuando la comparación implicaba variables continuas, se utilizó un t-test no pareado, mientras que el coeficiente de correlación de Pearson (r) fue calculado con el fin de medir las asociaciones entre variables continuas. Se adoptó el nivel p < 0.05 como punto de referencia para valorar aquellos resultados que eran estadísticamente significativos.

\section{Resultados}

\section{A. El cuestionario}

Un total de 1.020 personas (el 76,8\%) marcaron las casillas de "ningún problema" o "problemas leves" con respecto al humor depresivo en el cuestionario. 238 individuos (el 17,9\%) marcaron las de "problemas de cierta importancia", y 42 (el 3,2\%) los de "problemas graves" o "problemas muy graves". Estos datos no fueron cumplimentados por 28 personas (el 2,1\%). Aquellas personas que respondieron "problemas graves o muy graves" con respecto al humor depresivo eran algo más jóvenes que el resto, 65,0 +/- 6,5 años vs. 67,8 +/- 5,9 años $(\mathrm{t}=2,5 ; \mathrm{p}<0,05) . \mathrm{El} 46,4 \%$ vs. el $13,9 \%$ $(+/-=23,4 ; p<0,001)$ normalmente no estaban casadas (es decir, eran solteras, divorciadas, separadas o viudas) y, el 50,0\% vs. $13,5 \%(+/-=29,7 ; p<0,001)$ con frecuencia vivían solas. 


\section{B. Entrevistas clínicas}

De las 42 personas que fueron elegidas para ser entrevistadas, 4 (el 9,5\%) habían muerto antes de que la entrevista tuviese lugar, por tanto se entrevistó a 28 (el 73,7\%).
Las razones que adujeron aquellas personas que no participaron aparecen en la tabla I. Como se puede observar en la tabla II, se entrevistó a 13 hombres y a 15 mujeres aunque no se especificaron diferencias de sexo en cuanto a su humor depresivo.

Tabla I

Número de sujetos que están o no de acuerdo con participar

\begin{tabular}{lc} 
Razón para no participar & $(\mathrm{n}=42)$ \\
\hline Muerte & 4 \\
Rechazo: & 10 \\
Debido a enfermedad & 3 \\
Debido a enfermedad de familiar & 2 \\
No interesada & 5 \\
De acuerdo en participar & 28 \\
\hline
\end{tabular}

Tabla II

Características de los sujetos entrevistados que presentan afecto deprimido $(\mathrm{n}=28)$.

\begin{tabular}{lc} 
Variable & $\mathrm{n}(\%)$ \\
\hline Edad en años (media \pm SD) & $65.0 \pm 6.5$ \\
Sexo & \\
$\quad$ Hombres & $13(46.4)$ \\
$\quad$ Mujeres & $15(53.6)$ \\
Estado marital & \\
$\quad$ Casado & $15(53.6)$ \\
$\quad$ No casado ${ }^{1}$ & $13(46.4)$ \\
Plan de vida & \\
$\quad$ Vive con alguien más & $14(50.0)$ \\
$\quad$ Vive solo & $14(50.0)$ \\
Herencia de trastornos del humor & $6(21.4)$ \\
Diagnóstico DSM-III-R & $21(75.0)$ \\
$\quad$ Trastorno del humor & \\
$\quad$ Trastorno de ansiedad & $14(500)$ \\
Otros & $2(7.1)$ \\
Puntuación HAD-D (media \pm SD) & $10.3 \pm 4.1$ \\
Puntuación HAD-A (media \pm SD) & $11.5 \pm 3.3$ \\
Puntuación MADRS (media \pm SD) & $12.5 \pm 4.6$ \\
Puntuación GAF (media \pm SD) & $66 \pm 7$ \\
\hline
\end{tabular}

( $\left.{ }^{1}\right)$ Soltero, divorciado, separado o viudo.

${ }^{2}$ ) Incluyendo depresión diagnósticada/tratada.

\section{Trastornos psiquiátricos}

Todos, excepto una persona, cumplían los criterios DSM-III-R para un diagnóstico en el Eje I y 21 individuos (el 75\%) cumplieron criterios para trastornos afectivos o depresión bajo tratamiento (tabla III). 4 indivi- duos (el 14,3\%) cumplieron los criterios para un episodio depresivo mayor. La subcategoría más común de los trastornos afectivos fue la distimia, 9 personas (el $32,1 \%$ ). Se descubrió que en 6 individuos (el 21,4\%) se daban trastornos afectivos con bases probablemente hereditaria y que la dependen- 
Tabla III

Prevalencia de trastornos DSM-III-R entre los sujetos entrevistados $(\mathrm{n}=28)$

\begin{tabular}{lccc} 
Trastornos & Total $(\mathrm{n}=28)$ & Hombres $(\mathrm{n}=13)$ & Mujeres $(\mathrm{n}=15)$ \\
\hline Trastornos de humor & 21 & 8 & 13 \\
$\quad$ Depresión mayor & 4 & 1 & 3 \\
$\quad$ Depresión en tratamiento & 8 & 3 & 5 \\
$\quad$ Distimia & 9 & 4 & 5 \\
Trastornos de ansiedad & 14 & 6 & 8 \\
$\quad$ Trastorno de pánico & 3 & 1 & 2 \\
$\quad$ Trastorno de estrés postraumático & 1 & 1 & 0 \\
$\quad$ Trastorno de ansiedad generalizada & 10 & 4 & 6 \\
Abuso/dependencia alcohólica & 2 & 2 & 0 \\
No trastorno DSM-III-R & 1 & 1 & 0 \\
\hline
\end{tabular}

cia/abuso del alcohol también mostraba factores hereditarios en 4 personas (el 14,3\%). En cuanto al curso de la depresión, 11 de los individuos (el 52,4\%) que padecían un trastorno afectivo DSM-III-R o una depresión diagnosticada/tratada habían recibido antidepresivos debido a su enfermedad con anterioridad.

Se diagnosticaron trastornos de ansiedad en 14 personas (el 50,0\%) y la subcategoría más común fue la del trastorno de ansiedad generalizado, en 10 personas (el 35,7\%). Se descubrió una dependencia/abuso del alcohol en 2 personas (el 7,1\%). En consecuencia, observamos que había casos en los que existían trastornos afectivos junto con los de ansiedad. Las 9 personas (el 42,9\%) que padecían trastornos del humor, sufrían además trastornos afectivos y los 9 (el 64,3\%) que presentaban trastornos de ansiedad también mostraban trastornos afectivos.

\section{Gravedad de la depresión}

La puntuación media HAD-D de las personas que fueron entrevistadas fue 10,3 +/4,1 . El criterio que indica una posible depresión (una puntuación de 8 o superior) fue superado por 20 individuos (el 71,4\%), de los cuales 13 (el 46,4\%) habían obtenido puntuaciones por encima de 11 , que era un criterio definitivo de depresión. Según el
HAD-A, 25 personas (el 89,3\%) padecían un tipo de ansiedad posible o definitiva y en 19 (el 67,9\%) la ansiedad era definitiva. La puntuación media MADRS fue 12,5 +/- 4,6 y la correlación entre las puntuaciones HAD-D y MADRS mostró una $\mathrm{r}=0,72$ (fig.1).

\section{Nivel de funcionamiento}

Las puntuaciones GAF medias de las 28 personas entrevistadas fueron $66+/-7$ (rango de 55 a 85), con las proporciones más bajas en personas que padecían de trastornos del humor como de ansiedad, 62 +/- 5 (rango de 55 a 70), aunque la diferencia no fue estadísticamente significativa.

\section{Discusión}

El descubrimiento más importante de este estudio es el de que una clara mayoría de personas (el 75\%) que demostraron ser conscientes de su humor depresivo mediante un cuestionario, padecían en realidad de un trastorno afectivo según los criterios DSM-III-R o de una depresión bajo tratamiento, en el momento en el que fueron entrevistadas.

El valor de este estudio reside en el hecho de que estaba basado en un análisis de la pobla- 


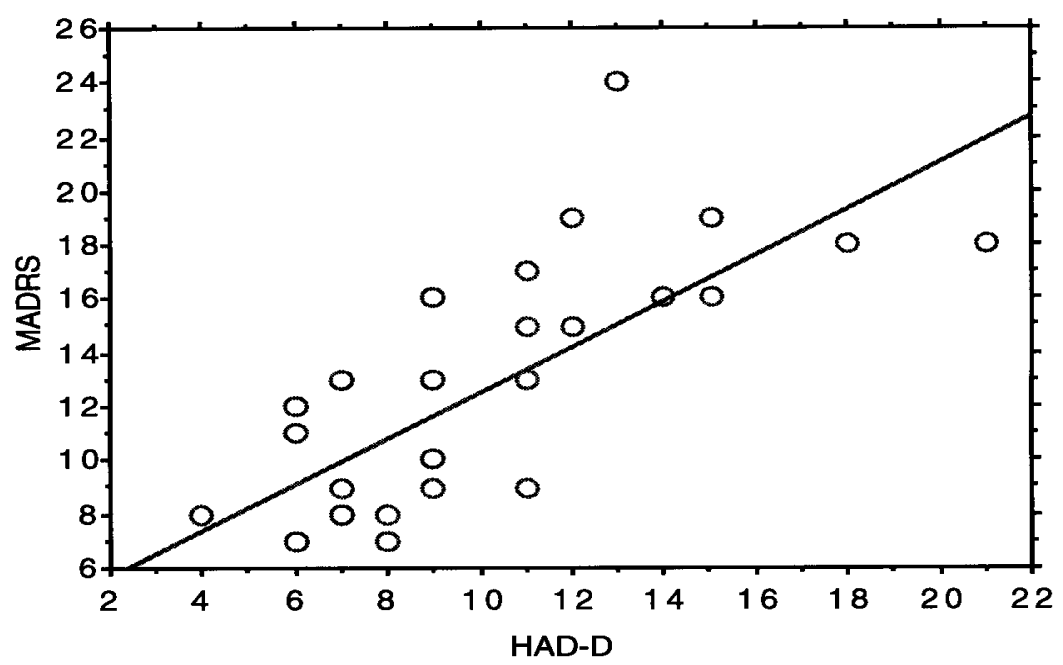

Figura 1. Valoración HAD-D y MADRS en sujetos entrevistados $(\mathrm{n}=28)$. Coeficiente de correlación Pearson productomomento $(\mathrm{r})=0,72$.

ción. La proporción de respuestas obtenidas mediante el cuestionario fue alto (el 74,1\%) y las personas que respondieron eran representativas de dicha población en lo que al sexo y a la edad se refiere (Statistic Yearbook 1996). La proporción de respuestas en cuanto a la entrevista también fue alta (el 73,7\%), sin embargo, el número de individuos que fueron entrevistados no fue tan elevado ( 28 personas), lo que indica que es necesario ser precavidos a la hora de interpretar los resultados.

Los datos subjetivos son importantes ya que la valoración y el tratamiento de la depresión se basa principalmente en los testimonios de los pacientes. Los instrumentos de estudio desarrollados con el fin de identificar a la depresión no valoran todos los criterios clínicos, sino que enfatizan diferentes aspectos de la depresión como por ejemplo la cognición, los síntomas somáticos, el comportamiento y las actitudes (Snaith 1993). Las escalas de autovaloración que están basadas en la sintomatología depresiva, incluyendo las causas físicas de estos síntomas, pueden dar lugar a una sobrestimación de la depresión, mientras que los instrumentos que siguen criterios estrictos pueden dar lugar a una infravaloración (Newman 1989). También se ha dicho que es menos probable que las personas de edad avanzada hablen de síntomas depresivos (Lyness et al. 1995) aunque según otros estudios, los ancianos presentan síntomas de este tipo con la misma frecuencia que las personas más jóvenes, pero en la mayoría de las ocasiones atribuyen su existencia a sus condiciones físicas (Knäuper \& Wittchen 1994). Además, aún no se ha probado que una pronta detección de la depresión tenga efectos positivos sobre los resultados obtenidos por los pacientes (Williams et al. 1999).

De las personas que contestaron el cuestionario, el 3,2\% admitieron tener humor depresivo. En una revisión posterior sobre el predominio de la depresión, Beekman et al. (Beekman et al. 1999) descubrió que su prevalencia variaba entre el 0,4\% y el 10,2\% y que todos los estudios excepto uno, de un total de 16, aportaban niveles de prevalencia de Depresión Mayor que estaban por debajo del $5 \%$. 
Se podría decir que los niveles de prevalencia no están lo suficientemente valorados debido a una mayor representación de casos depresivos en aquellas personas que no contestaron el cuestionario. Sin embargo, otros estudios no han encontrado un mayor predominio de casos depresivos comparado con los que muestran las personas que contestaron el cuestionario (Kivelä et al. 1988; Livingston et al. 1990).

Existe un gran número de instrumentos disponibles para estudiar las depresiones. En un trabajo sobre el estudio de la depresión, Schade et al. concluía que en este tipo de análisis, "menos es más". Esta afirmación está basada en el hecho de que los "instrumentos abreviados" funcionan tan bien como los cuestionarios completos (Schade et al. 1998). El valor de los resultados utilizando una sola pregunta sobre la depresión, ya se había apreciado en pacientes de Atención Primaria (Williams et al. 1999, Berwick et al. 1991), así como en otros pacientes (Mahoney et al. 1994), en enfermos terminales (Chochinov et al. 1997) y en pacientes con trastorno de ansiedad (McKenzie \& Marks 1999).

Los criterios comunes de la depresión, según el sistema DSM, es el humor depresivo y la anhedonia (o pérdida de interés por cualquier actividad). Un instrumento de investigación que tenga en cuenta dos preguntas (depresión y anhedonia) presenta características similares a otros instrumentos de valoración de la depresión de los que se utilizan en pacientes adultos; así, una respuesta negativa a estas dos preguntas hace que la depresión no sea probable (Whooley \& Browner 1998). Además, una entrevista compuesta por una sola pregunta sobre el humor depresivo supera tanto al cuestionario como a una medida visual análoga en pacientes terminales (Chochinov et al. 1997). El hecho de añadir una pregunta sobre la anhedonia no mejoró la precisión del diag- nóstico, lo que hace pensar que una sola pregunta sobre la depresión es suficiente.

En este estudio también utilizamos la escala HAD-D que señala a la anhedonia como el elemento más importante de la depresión. Se descubrió que el 71,4\% de las personas que afirmaban padecer humor depresivo, presentaban una depresión posible o concreta según los criterios HAD-D, lo que demostraba la gran coincidencia entre los autodiagnósticos y las valoraciones realizadas por los expertos sobre depresión. Estos resultados indican que una gran parte de los individuos que evaluaron su propio trastorno afectivo también padecían de una anhedonia sustancial, cumpliendo por tanto los dos criterios principales que se necesitan para diagnosticar una depresión.

La subcategoría más común dentro de los trastornos afectivos fue la distimia y, cerca de la mitad de las personas que padecían este tipo de trastorno habían sido tratadas con anterioridad por un síndrome depresivo, lo que mostraba que muchas de ellas padecían más de síntomas depresivos permanentes o recurrentes que de episodios depresivos breves o transitorios.

Nuestros resultados muestran que existe una superposición considerable entre los trastornos de ansiedad y la depresión. De entre las personas que presentaron un humor depresivo, el 89,3\% sobrepasaban el punto de corte marcado por el HAD-A, indicando la existencia de una ansiedad que podía ser posible o concreta y el $50 \%$ padecían trastornos de ansiedad cuando fueron entrevistados. Los estudios anteriores también habían descubierto proporciones altas de comorbilidad de depresión y ansiedad en ancianos (Flint 1994); y también se ha señalado como la depresión en estas personas forma parte de un síndrome que contiene sintomatología ansiosa y depresiva (Gottfries 1998). 
El bajo nivel de actividad calculado mediante la Escala de Valoración del Funcionamiento (GAF), implica que la aparición de humor depresivo lo disminuye. Incluso los niveles moderados de una depresión,según indican las puntuaciones de MADRS, están relacionados con discapacidades. Estos resultados, aunque con ciertos límites, muestran como una sola pregunta sobre trastornos afectivos es clínicamente importante en estudios comunitarios de personas con edad avanzada. La realidad es que se detectaron a personas que presentaban síntomas permanentes o recurrentes con un limitado nivel de funcionamiento y con síntomas de ansiedad. No debería considerarse como un criterio definitivo,pero una respuesta afirmativa puede ser utilizada para determinar qué personas necesitan de estudios psiquiátricos más profundos o de otro tipo con el fin de alcanzar un diagnóstico mas seguro y preciso.

\section{Agradecimientos}

Este estudio ha sido financiado por el Instituto de Investigaciones Médicas sueco (Proyecto 06869), por el Instituto de Investigación Dalarna, por la Asociación Sueca de Psiquiatría, por la Fundación Marta y Nicke Nasvells, por el Fondo Bror Gadelius Memory, por la Fundación Gun y Bertil Stohnes y por la Fundación Sueca de Lundbeck.

\section{Bibliografía}

ALLEN, N.,AMES, D.,ASHBY, D., BENNETTS, K., TUCKWELL, V., WEST, C. A brief sensitive screening instrument for depression in later life. Age and Ageing, 23, 213-219, 1994

ALMEIDA, O., ALMEIDA, S. Short versions of the geriatric depression scale: a study of their validity for the diagnosis of a major depressive episode according to ICD10 and DSM-IV. International Journal of Geriatric Psy chiatry, 14, 858-865, 1999.

ANDERSEN, E., MALMGREN, J., CARTER, W., PATRICK, D. Screening for depression in well older adults: evaluation of a short form of the CES-D (Center for Epidemiologic Studies Depression Scale). American Jour nal of Preventive Medicine, 10, 77-84, 1994.

APA Diagnostic and Statistical Manual of Mental Disorders, Third edition, revised (DSM-III-R), Washington DC: American Psychiatric Association, 1987.

BEEKMAN, A.,COPELAND, J.,PRINCE,M. Review of community prevalence of depression in later life. British Journal of Psychiatry, 174, 307-311, 1999.

BERWICK, D., MURPHY, J., GOLDMAN, P., WARE, J.J., BARSKY, A., WEINSTEIN, M. Performance of a five-item mental health screening test. Medical Care, 29, 169-176, 1991.

BLAZER, D., HUGHES, D., GEORGE, L. The epidemiology of depression in an elderly community population. Gerontologist, 27, 281-287, 1987.

CHOCHINOV, H., WILSON, K., ENNS, M., LANDER, S. "Are you depressed?"Screening for depression in the terminally ill. American Journal of Psychiatry, 154, 674-676, 1997.

FLINT, A.J. Epidemiology and comorbidity of anxiety disorders in the elderly. American Journal of Psychiatry, 151, 640-649, 1994.

GOTTFRIES, C. Is there a difference between elderly and younger patients with regard to the symptomatology and aetiology of depression? International Clinical Psychpharmacology, 13 (suppl 5), S13-S18, 1998.

GURLAND, B. The comparative frequency of depression in various adult age groups. Journal of Gerontology, 31, 283-292, 1976.

HENDERSON, A., JORM, A., MACKINNON, A., CHRISTENSEN, H., SCOTT, L., KORTEN, A., DOYLE, $\mathrm{C}$. The prevalence of depressive disorders and the distribution of depressive symptoms in later life: a survey using Draft ICD-10 and DSM-III-R. Psychological Medicine, 23, 719-729, 1993.

HOYL, M.,ALESSI, C., HARKER, J., JOSEPHSON, K.,PIETRUSZKA, F., KOELFGEN, M.,MERVIS, J.,FITTEN, L., RUBENSTEIN, L. Development and testing of a five-item version of the Geriatric Depression Scale. Jour nal of the American Geriatric Society, 47, 873-878,1999.

IRWIN, M., ARTIN, K., OXMAN, M. Screening for depression in the older adult: criterion validity of the 10item Center for Epidemiological Studies Depression scale 
(CES-D). Archives of Internal Medicine, 159, 1701-1704, 1999.

KANOWSKI, S. Age-dependent epidemiology of depression. Gerontology, 40 (suppl 1), S1-S4, 1994.

KIVELÄ, S.L., PAHKALA, K., LAIPPALA, P. Prevalence of depression in an elderly population in Finland. Acta Psychiatrica Scandinavica, 78, 401-413, 1988.

KNÄUPER, B.,WITTCHEN, H. Diagnosing major depression in the elderly: evidence for response bias in standardized diagnostic interviews? Journal of Psychiatric Research, 28, 147-164, 1994.

LIVINGSTON, G., HAWKINS, A., GRAHAM, N., BLIZARD, B., MANN, A. The Gospel Oak Study: prevalence rates of dementia, depression and activity limitation among elderly residents in Inner London. Psychological Medicine, 20, 137-146, 1990.

LYNESS, J., COX, C., CURRY, J., CONWELL, Y., KING, D.,CAINE,E. Older age and the underreporting of depressive symptoms. Journal of the American Geriatric Society, 43, 216-221, 1995.

MAHONEY, J., DRINKA, T.,ABLER, R., GUNTERHUNT, G.,MATTHEWS, C., GRAVENSTEIN, S., CARNES, M. Screening for depression: single question versus GDS. Journal of the American Geriatric Society 42, 10061008, 1994.

McKENZIE, N.,MARKS, I. Quick rating of depressed mood in patients with anxiety disorders. British Journal of Psychiatry, 174, 266-269, 1999.

MONTGOMERY, S.,ASBERG, M. A new depression scale designed to be sensitive to change. British Journal of Psychiatry, 134, 382-389, 1979.

MURPHY, E.,SMITH,R.,LINDESAY, J.,SLATTERY, J. Increased mortality rates in late-life depression. British Journal of Psychiatry, 152, 347-353, 1988.

NEWMAN, J. Aging and depression. Psychology and Aging, 4, 150-165, 1989.

PALSSON, S.,SKOOG, I. The epidemiology of affective disorders in the elderly:a review. International Clinical Psychopharmacology, 12 (suppl 7), S3-S13,1997.

ROBERTS, R., KAPLAN, G., SHEMA, S., STRAWBRIDGE, W. Does growing old increase the risk for depression? American Journal of Psychiatry, 154, 13841390, 1997.
SCHADE, C., JONES, E. J., WITTLIN, B. A ten-year review of the validity and clinical utility of depression screening. Psychiatric Services, 49, 55-61, 1998.

SKOOG, I.,NILSSON, L.,LANDAHL, S.,STEEN, B. Mental disorders and the use of psychotropic drugs in an 85-year old urban population. International Psychogeria trics, 5, 33-48, 1993.

SNAITH, P. What do depression rating scales measure? British Journal of Psychiatry, 163, 293-298, 1993.

SPITZER,R.,WILLIAMS, J.,GIBBON, M.,FIRST, M. The Structured Clinical interview for DSM-III-R (SCID). I: History, rationale, and description. Archives of General Psychiatry, 49, 624-629, 1992.

STATISTISK ÅRSBOK. Swedish Central Bureau of Statistics, Yearbook 1995, Stockholm, 1996.

VALLIANT, G.,ORAV, J.,MEYER, S., VALLIANT, L. M., ROSTON, D. Late-life consequences of affective spectrum disorder. International Psychogeriatrics, 8, 12-32, 1996.

WEYERER, S., KILLMANN, U.,AMES, D.,ALLEN, N. The Even Briefer Assessment Scale for Depression (EBAS DEP):its suitability for the elderly in geriatric care in English- and German-speaking countries. International Journal of Geriatric Psychiatry, 14, 743-780, 1999.

WHOOLEY, M.,BROWNER, W. Associations between depressive symtoms and mortality in older women. Study of Osteoporotic Fractures Research Group. Archives of Internal Medicine, 158, 2129-2135, 1998.

WILliAMS, J.J., MULROW, C., KROENKE, K., DHANDA,R., BADGETT, R.,OMORI, D.,LEE, S. Casefinding for depression in primary care:a randomized trial. American Journal of Medicine, 106, 36-43, 1999.

ZIGMOND, A.S., SNAITH, R.P. The Hospital Anxiety and Depression Scale. Acta Psychiatrica Scandinavica,67, 361-370, 1983.

Dirección para correspondencia:

Lena Mallon, MD, PhD

Sleep Disorders Unit

Department of Neuroscience, Psychiatry

University Hospital

SE-751 85 Uppsala

SWEDEN 